\title{
Off-axis vortices in trapped Bose-condensed gases: Angular momentum and frequency splitting
}

\author{
Montserrat Guilleumas and Robert Graham \\ Fachbereich Physik, Universität Gesamthochschule Essen, 45117 Essen, Germany
}

(Received 18 December 2000; published 14 August 2001)

\begin{abstract}
We consider noncentered vortices and their arrays in a cylindrically trapped Bose-Einstein condensate at zero temperature. We study the kinetic energy and the angular momentum per particle in the Thomas-Fermi regime and their dependence on the distance of the vortices from the center of the trap. Using a perturbative approach with respect to the velocity field of the vortices, we calculate, to first order, the frequency shift of the collective low-lying excitations due to the presence of an off-center vortex or a vortex array, and compare these results with predictions that would be obtained by the application of a simple sum-rule approach, previously found to be very successful for centered vortices. It turns out that the simple sum-rule approach fails for off-centered vortices.
\end{abstract}

DOI: 10.1103/PhysRevA.64.033607

PACS number(s): 03.75.Fi, 05.30.Jp, 32.80.Pj, 67.40.Db

\section{INTRODUCTION}

Vortices in trapped Bose-condensed gases have recently been observed at JILA [1] and at ENS [2,3]. At ENS they have been formed by stirring the condensate with a focused laser beam with an angular frequency $\Omega$. Experimentally there exists a threshold of the angular frequency of the stirring beam $\left(\Omega_{c}\right)$ to nucleate a single vortex. When $\Omega \sim \Omega_{c}$, one vortex is created at the stable position at the center of the trap. But at $\Omega>\Omega_{c}$, and depending on the frequency of the stirring beam, it is possible to create configurations with different number of vortices forming vortex arrays that are stable compared to a single vortex with correspondingly larger circulation.

The presence of vortices in trapped condensates has been revealed by time-of-flight analysis $[1,2]$ and recently, by exciting the quadrupole oscillations of a stirred Bose-Einstein condensate [3] and observing their frequency splitting. With this method it has also been possible to measure the angular momentum induced by the rotation of the trap. Direct measurement of the angular momentum is difficult in atomic gases, since most of the diagnostic techniques provide information on the density profiles. As expected, for stirring frequencies below $\Omega_{c}$, no angular momentum is observed [3], since no vortex can be nucleated, and just above $\Omega_{c}$, the angular momentum has a jump of $\sim \hbar$, which indicates the presence of a stable singly quantized vortex at the center of the trap. However, for higher stirring frequencies, an a priori unexpected behavior of the angular momentum has been experimentally found [3]: for $\Omega>\Omega_{c}$, when vortex arrays are nucleated, the measured angular momentum does not show any jump of size $\hbar$ as a new singly quantized vortex is nucleated, as is, e.g., observed for the corresponding experiment in superfluid helium [4]; instead it is a smooth and increasing function of $\Omega$. We shall see that this is not in contradiction with the quantization of the circulation of a vortex, but it is a consequence of the nucleation of off-axis vortices that form a vortex array.

The purpose of the present paper is to provide a detailed analysis of the dependence of the kinetic energy and the angular momentum on the distance of an off-centered vortex with respect to the symmetry axis. We will generalize the results to vortex arrays to give a physical insight of the smooth behavior of the angular momentum found experimentally.

In an axially symmetric trap the axial component of angular momentum $(m)$ is a good quantum number, and in the absence of vortices, the collective excitations of the condensate are degenerate with respect to the sign of $m$. However, the presence of vortices breaks this degeneracy, and elementary excitations carrying opposite angular momentum are no longer degenerate. The frequency shifts of the quadrupole oscillations due to the presence of a vortex on the symmetry axis of the trap, have been calculated in Ref. [5] using a sum-rule approach. Analytic results for the energy splitting are obtained in Ref. [5] in the Thomas-Fermi (TF) limit for the quadrupole modes and have been used in Ref. [3] to measure the angular momentum of the condensate. General expressions based on the hydrodynamic approach for the energy splitting due to a centered vortex have been obtained in Refs. [6,7] within perturbation theory.

In this paper we consider off-centered quantized vortex lines in large cylindrically confined condensates at zero temperature, and therefore, no dissipation mechanism [8] is taken into account. We calculate the kinetic energy and the angular momentum with respect to the symmetry axis $[9,10]$ of an array of vortex lines in a trapped Bose-condensed gas. Using the perturbative approach proposed in Ref. [7], and assuming the Thomas-Fermi limit, we generalize the analytic expressions for the energy splitting of the low-lying collective modes to consider the effect of the distance of offcentered vortices with respect to the symmetry axis. We also compare with the results obtained by a direct extension of the sum-rule approach to off-center vortices, but find that such an extension, at least in the direct version examined here, fails.

\section{VORTEX STATES}

We consider a weakly interacting Bose-condensed gas confined in a harmonic trap $V_{\text {ext }}(\mathbf{r})$ at zero temperature. The condensate wave function can be written in terms of its density $\rho(\mathbf{r})=|\Psi(\mathbf{r})|^{2}$ and phase $S(\mathbf{r})$ by

$$
\Psi(\mathbf{r})=\sqrt{\rho(\mathbf{r})} \exp [i S(\mathbf{r})] .
$$


The number of atoms in the condensate is $\int d \mathbf{r}|\Psi|^{2}=N$, and the superfluid velocity is given by $\mathbf{v}=(\hbar / M) \nabla S$, where $M$ is the atomic mass. The ground-state wave function in the absence of vortices has a spatially constant phase and therefore zero velocity, but when a quantized vortex is present, the phase and the velocity field of the ground state have to be determined according to the boundary conditions of the system.

\section{A. Condensate density}

The ground-state wave function $\Psi(\mathbf{r})$ is a stationary solution of the Gross-Pitaevskii equation

$$
\left(-\frac{\hbar^{2} \nabla^{2}}{2 M}+V_{\mathrm{ext}}(\mathbf{r})+g|\Psi(\mathbf{r})|^{2}\right) \Psi(\mathbf{r})=\mu \Psi(\mathbf{r}),
$$

where the coupling constant $g$ is given by the $s$-wave scattering length $a$ through $g=4 \pi \hbar^{2} a / M$, and $\mu$ is the chemical potential fixed by the normalization condition of the ground state.

Nonrotating experimental traps [1-3] have axial symmetry, with different radial $\left(\omega_{\perp}\right)$ and axial $\left(\omega_{z}\right)$ trapping frequencies, whose ratio defines the anisotropy parameter $\lambda$ $=\omega_{z} / \omega_{\perp}$. So far, vortex arrays have been produced at ENS $[2,3]$ in a highly anisotropic cigar-shaped trap (quasicylindrically symmetric) with $\lambda \sim 0.05$. Thus, for simplicity, we consider an idealized cylindrical trap that is uniform in the $z$ direction $\left(\omega_{z}=0\right)$ and has a harmonic confining potential in the radial direction of the form

$$
V_{\mathrm{ext}}\left(r_{\perp}\right)=\frac{1}{2} M \omega_{\perp}^{2} r_{\perp}^{2},
$$

with $r_{\perp}^{2}=x^{2}+y^{2}$. The harmonic trap frequency $\omega_{\perp}$ provides a typical length scale for the system $a_{\perp}=\left(\hbar / M \omega_{\perp}\right)^{1 / 2}$.

We consider the Thomas-Fermi regime [11], valid for large condensates with positive scattering length, where the kinetic pressure can be neglected compared to the interaction energy density. The ground-state density of the condensate, in the absence of vortices, is given by

$$
\rho_{0}\left(r_{\perp}\right)=\frac{\mu}{g}\left(1-\frac{r_{\perp}^{2}}{R_{\perp}^{2}}\right)
$$

for $r_{\perp} \leqslant R_{\perp}$ and $\rho_{0}\left(r_{\perp}\right)=0$ elsewhere. The Thomas-Fermi radius of the cylindrical condensate is $R_{\perp}=\left(2 \mu / M \omega_{\perp}^{2}\right)^{1 / 2}$. The density is defined in the interval $-R_{z} \leqslant z \leqslant R_{z}$, where $2 R_{z}$ is the length of the cylinder. In the cylindrical geometry, the validity of the Thomas-Fermi approximation for the ground state is guaranteed by the condition $N a /\left(2 R_{z}\right)$ $=N_{\perp} a \gg 1$, or equivalently $\mu \gg \hbar \omega_{\perp}$, where $N_{\perp}=N /\left(2 R_{z}\right)$ is the number of atoms per unit length [12]. From Eq. (4),

$$
N_{\perp}=\int_{0}^{R_{\perp}} \rho_{0} 2 \pi r_{\perp} d r_{\perp}=\frac{\mu}{g} \frac{\pi R_{\perp}^{2}}{2} .
$$

When a quantized vortex is present at the position $\mathbf{r}_{\perp 0}$, the density of the system drops to zero at the center of the vortex core whose size is determined by the healing length $\xi$. In the limit of large systems it can be approximated by $\xi$ $=\left[8 \pi \rho_{0}\left(\mathbf{r}_{\perp 0}\right) a\right]^{-1 / 2}$, where $\rho_{0}\left(\mathbf{r}_{\perp 0}\right)$ is the density of the condensate at the position of the vortex core but in the absence of vorticity. For a centered vortex, Eq. (4) yields $\rho_{0}(0)=\mu / g$, and the corresponding healing length $\xi_{0}$ $=\xi(0)$ can be rewritten as

$$
\frac{\xi_{0}}{R_{\perp}}=\left(\frac{a_{\perp}}{R_{\perp}}\right)^{2},
$$

implying the following set of TF inequalities for the length scales $\xi_{0} \ll a_{\perp} \ll R_{\perp}$. Analogously, from Eq. (4) the local healing length [12] is

$$
\xi\left(r_{\perp 0} / R_{\perp}\right)=\frac{\xi_{0}}{\sqrt{1-r_{\perp 0}^{2} / R_{\perp}^{2}}} .
$$

Within the TF approximation, analytical expressions for the density of a vortex state have been obtained for a centered vortex $[6,7]$ and for a straight off-axis vortex line [13]. However, in weighted spatial averages of quantities varying only on the scale $R_{\perp}$, the density of a vortex state may be replaced by the density of the vortex-free state, the corrections being only of order $\left(\xi / R_{\perp}\right)^{2}=\left(a_{\perp} / R_{\perp}\right)^{4}$ [7], which is negligible.

\section{B. Velocity field}

We consider states having a quantized vortex line along the $z$ axis and all the atoms flowing around it with quantized circulation. The property of a single-valued wave function leads to the quantization of the circulation around an arbitrary closed loop that encloses the vortex core

$$
\mathcal{K}=\oint \mathbf{v} \cdot d \mathbf{l}=\frac{\hbar}{M} \oint \boldsymbol{\nabla} S \cdot d \mathbf{l}=\frac{h \kappa}{M},
$$

where the integer number $\kappa$ is the quantum of circulation. For sufficiently large frequencies $\Omega>\Omega_{c}$, a single vortex line with an integer quantum number $\kappa>1$ can, in principle, appear, but this state is unstable and fragments into a vortex array formed by $\kappa$ vortices, each with a unit of circulation and position $\mathbf{r}_{\perp 0 i}(1 \leqslant i \leqslant \kappa)$ relative to the symmetry axis.

We will not consider large arrays of vortex lines, where an average vortex density can be defined $[4,8,9]$, but small ones, corresponding to the experimental region [2,3] of multiple vortices $(\kappa \leqslant 5)$. Large vortex arrays may enter the "turbulent" region found experimentally. For small vortex arrays the density of the system can be approximated by the density of a vortex-free condensate, because vortices rotating in the same direction experience an effective repulsive interaction [4], and the inhomogeneities due to the well-separated vortex cores are negligible in the condensate density $\left(\xi \ll R_{\perp}\right)$.

Let us assume a generic quantized vortex line parallel to the $z$ axis at the position $\mathbf{r}_{\perp 0}$ and with quantized circulation $\kappa$. Then, from Eq. (8), $\boldsymbol{\nabla} \times \mathbf{v}=(h \kappa / M) \delta\left(\mathbf{r}_{\perp}-\mathbf{r}_{\perp 0}\right) \hat{\mathbf{z}}$, where $\hat{\mathbf{z}}$ is the unit vector in the $z$ direction, and the superfluid is irrotational everywhere except for the vortex core at $\mathbf{r}_{\perp}$ $=\mathbf{r}_{\perp 0}$, where the density vanishes. 
For a positively oriented vortex in an infinite, uniform system, the velocity then is

$$
\mathbf{v}_{\text {uni }}\left(\mathbf{r}_{\perp}\right)=\frac{\hbar \kappa}{M} \frac{\hat{\mathbf{z}} \times\left(\mathbf{r}_{\perp}-\mathbf{r}_{\perp 0}\right)}{\left|\mathbf{r}_{\perp}-\mathbf{r}_{\perp 0}\right|^{2}} .
$$

In particular, for a centered vortex only the tangential component carries nonvanishing atomic flux, and $\mathbf{v}$ $=\hbar \kappa /\left(M r_{\perp}\right) \hat{\boldsymbol{\varphi}}$, where $\hat{\boldsymbol{\varphi}}$ is the unit vector in the tangential direction in cylindrical coordinates $\left(r_{\perp}, \varphi, z\right)$. Equation (9) once again shows that a cutoff of the order of the local healing length $\xi$ for the distance $\left|\mathbf{r}_{\perp}-\mathbf{r}_{\perp 0}\right|$ is needed, because at distances smaller than $\sqrt{2} \xi$, the velocity (9) surpasses the local velocity of sound $c_{s}\left(\mathbf{r}_{\perp}\right)=\sqrt{g\left|\psi\left(\mathbf{r}_{\perp}\right)\right|^{2} / M}$.

For a confined system, the velocity field is affected by the boundary of the system and by the spatially varying density, and has to fulfill the following physical conditions: (i) the normal velocity has to vanish at the boundary, and (ii) the condition for stationary flow $\boldsymbol{\nabla} \cdot(\rho \mathbf{v})=0$ has to be satisfied. It is well known from the rotating bucket experiment in superfluid helium $[9,10,12]$ that for an homogeneous system confined in a cylinder of radius $R_{\perp}$, the normal velocity vanishes at the boundary by introducing an oppositely oriented image vortex at $\mathbf{r}_{\perp 1}=\left(R_{\perp} / r_{\perp 0}\right)^{2} \mathbf{r}_{\perp 0}$. The resulting velocity field is

$$
\mathbf{v}_{0}\left(\mathbf{r}_{\perp}\right)=\frac{\hbar \kappa}{M} \frac{\hat{z} \times\left(\mathbf{r}_{\perp}-\mathbf{r}_{\perp 0}\right)}{\left|\mathbf{r}_{\perp}-\mathbf{r}_{\perp 0}\right|^{2}}-\frac{\hbar \kappa}{M} \frac{\hat{z} \times\left(\mathbf{r}_{\perp}-\mathbf{r}_{\perp 1}\right)}{\left|\mathbf{r}_{\perp}-\mathbf{r}_{\perp 1}\right|^{2}} .
$$

If the system has a density gradient, the condition (ii) for stationary flow is fulfilled by introducing a small correction to the velocity, which can be neglected when the density varies over a larger scale than the healing length [12]. Thus, in the Thomas-Fermi limit, the velocity field can be approximated as $\mathbf{v} \simeq \mathbf{v}_{0}$.

A vortex at $\mathbf{r}_{\perp 0}$ is influenced by the velocity-field induced by its mirror vortex. If $\mathbf{r}_{\perp 0}$ approaches the boundary, this velocity diverges, signaling the break-down of the ThomasFermi approximation. A suitable lower cutoff for the distance from the boundary, $\xi_{\text {boundary }}=R_{\perp}\left(\xi_{0} / 2 R_{\perp}\right)^{2 / 3}$, is implied by restricting the velocities induced by the mirror vortices by the local velocity of sound.

From Eq. (10) one can see that the contribution of a vortex (vortex and image vortex) is additive. Therefore, the velocity field corresponding to a vortex array formed by $\kappa$ singly quantized vortices at $\mathbf{r}_{\perp 0 i}$ with $i=1, \ldots, \kappa$, is then

$$
\mathbf{v}\left(\mathbf{r}_{\perp}\right)=\sum_{i=1}^{\kappa} \mathbf{v}_{i}\left(\mathbf{r}_{\perp}\right)
$$

with

$$
\mathbf{v}_{i}\left(\mathbf{r}_{\perp}\right)=\left(\frac{\hbar}{M} \frac{\hat{z} \times\left(\mathbf{r}_{\perp}-\mathbf{r}_{\perp 0 i}\right)}{\left|\mathbf{r}_{\perp}-\mathbf{r}_{\perp 0 i}\right|^{2}}-\frac{\hbar}{M} \frac{\hat{z} \times\left(\mathbf{r}_{\perp}-\mathbf{r}_{\perp 1 i}\right)}{\left|\mathbf{r}_{\perp}-\mathbf{r}_{\perp 1 i}\right|^{2}}\right),
$$

where $\mathbf{r}_{\perp 1 i}=\left(R_{\perp} / r_{\perp 0 i}\right)^{2} \mathbf{r}_{\perp 0 i}$ is the position of the vortex image corresponding to the $i$-vortex.

\section{KINETIC ENERGY}

In the Thomas-Fermi limit the dominant part of the excess energy of a vortex state over the ground state without a vortex, is given by the kinetic energy of the velocity field [6]. It is obtained from Eqs. (11) and (12) in the form

$$
E_{k i n}=\sum_{i=1}^{\kappa} E_{i}+\sum_{i=1}^{\kappa} \sum_{j=1}^{i-1} E_{i j}
$$

with

$$
\begin{aligned}
E_{i}= & \frac{M}{2} \int_{-R_{z}}^{R_{z}} d z \int_{0}^{R_{\perp}} d r_{\perp} r_{\perp} \rho_{0}\left(r_{\perp}, z\right) \\
& \times \int_{0}^{2 \pi} d \varphi \mathbf{v}_{i}^{2}\left(r_{\perp}, z, \varphi\right), \\
E_{i j}= & M \int_{-R_{z}}^{R_{z}} d z \int_{0}^{R_{\perp}} d r_{\perp} r_{\perp} \rho_{0}\left(r_{\perp}, z\right) \\
& \times \int_{0}^{2 \pi} d \varphi \mathbf{v}_{i}\left(r_{\perp}, z, \varphi\right) \cdot \mathbf{v}_{j}\left(r_{\perp}, z, \varphi\right),
\end{aligned}
$$

where $E_{i}$ is the self-energy of the $i$ vortex and $E_{i j}$ the binary interaction energy between a couple of vortices $i$ and $j$. Let us define the dimensionless quantities $\widetilde{E}_{i}$ $=E_{i} /\left(2 N \hbar^{2} / M R_{\perp}^{2}\right)$, and analogously $\widetilde{E}_{i j}$ and $\widetilde{E}_{\text {kin }}$. For an array of vortex lines along the $z$ axis in the idealized cylindrical trap described by Eqs. (3) and (4), the integrals in Eqs. (14) and (15) can be performed and we obtain after some calculations, ${ }^{1}$ defining the rescaled distance vector of the vortex line from the $z$ axis $\mathbf{x}_{i}=\mathbf{r}_{\perp 0 i} / R_{\perp}$,

$$
\begin{aligned}
\widetilde{E}_{i}= & \frac{1-x_{i}^{4}}{2 x_{i}^{2}} \ln \left(1-x_{i}^{2}\right)+\left(1-x_{i}^{2}\right) \ln \left(\frac{R_{\perp}}{\sqrt{2} \xi_{0}}\right), \\
\widetilde{E}_{i j}= & \left(1-\mathbf{x}_{i} \cdot \mathbf{x}_{j}\right) \ln \left(\frac{1}{\left(\mathbf{x}_{i}-\mathbf{x}_{j}\right)^{2}}\right) \\
& +\left(1+\frac{1-x_{i}^{2}-x_{j}^{2}-x_{i}^{2} x_{j}^{2}}{2 x_{i}^{2} x_{j}^{2}} \mathbf{x}_{i} \cdot \mathbf{x}_{j}\right) \\
& \times \ln \left(1+x_{i}^{2} x_{j}^{2}-2 \mathbf{x}_{i} \cdot \mathbf{x}_{j}\right) \\
& -\frac{1-x_{i}^{2}-x_{j}^{2}+x_{i}^{2} x_{j}^{2}}{x_{i}^{2} x_{j}^{2}}\left|\mathbf{x}_{i} \times \mathbf{x}_{j}\right| \arctan \left(\frac{\left|\mathbf{x}_{i} \times \mathbf{x}_{j}\right|}{1-\mathbf{x}_{i} \cdot \mathbf{x}_{j}}\right) .
\end{aligned}
$$

\footnotetext{
${ }^{1}$ It is useful for this purpose to adopt a complex representation of the transverse vector $\mathbf{r}_{\perp} \rightarrow z=x+i y$ and of the velocity field of a vortex at $z_{0}$ in the form $\mathbf{v}_{j} \rightarrow v_{j}=(\hbar / M) i z /\left(r_{\perp}^{2}-z z_{0}^{*}\right)$ with complex conjugate $v_{j}^{*}=-(\hbar / M) i /\left(z-z_{0}\right)$, and to perform the integral over $\varphi$ at a fixed value of $r_{\perp}=|z|$ as a complex contour-integration making use of the residuum theorem.
} 


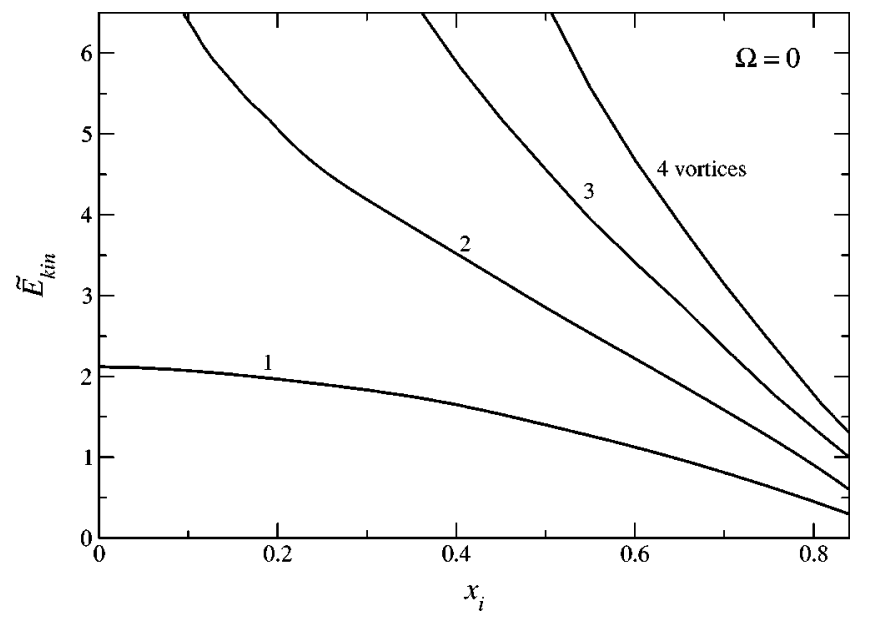

FIG. 1. Dimensionless energy of symmetrical vortex arrays of 1, 2,3 , and 4 singly quantized vortices (from bottom curve to top curve, respectively) as a function of the rescaled distance of the vortex lines from the $z$ axis. The condensate has $N=2.5 \times 10^{5}$ atoms of ${ }^{87} \mathrm{Rb}$ with $R_{\perp}=3.8 \mu \mathrm{m}$, which correspond to the experimental parameters of Ref. [3]

Here the healing length $\sqrt{2} \xi_{0} / R_{\perp}$ is used as a cutoff. It is of interest to remark that for $R_{\perp}$ and $\xi_{0}$ fixed, both $E_{i}$ and $E_{i j}$ are proportional to $N$. Expression (16) for the kinetic energy of a vortex in the presence of its image vortex (self-energy), agrees with the result presented by Fetter [14] for one vortex. The kinetic energy of an array of vortices without their images was calculated by Castin and Dum [15]. Due to the presence of image vortices, introduced to ensure the vanishing of the normal component of the velocity at the boundary, our result differs from that in Ref. [15], but it is only slightly more complicated.

In Fig. 1 we plot the kinetic energy of symmetrical arrays of singly quantized $1,2,3$, and 4 vortices, analogous to the experimental configurations of Ref. [3], as a function of the normalized distance from the trap axis. The kinetic energy goes to zero as the distance of the vortices from the boundary of the condensate goes to zero. This is a consequence of the boundary condition on the surface, where each vortex meets its image and thus is annihilated in the process. As mentioned in the preceding section, a lower cutoff must be imposed on the distance of the vortex line from the boundary. This is tantamount to restricting $x_{i}$ by $x_{i}<1-\left(\xi_{0} / 2 R_{\perp}\right)^{2 / 3}$. The energy is maximal at the center of the trap, where expression (17) diverges logarithmically unless a cutoff on the minimal distance of the vortex cores from the $z$ axis is imposed, whose size is again taken as $\sqrt{2} \xi_{0} / R_{\perp}$. Figure 1 makes it clear that a single vortex or a vortex array must be created at the boundary, where the energy required to set up the necessary velocity field, becomes vanishingly small. However, as we have already mentioned, the description of a vortex close to the boundary, is outside the scope of the present approach, because there the Thomas-Fermi approximation, on which it is based, breaks down.

\section{ANGULAR MOMENTUM}

Let us recall the expression of the angular momentum of a vortex state in an imperfect Bose gas $[9,10]$. We consider an axially symmetric condensate and are interested in the angular momentum around its symmetry axis defined along the $z$ axis. For a single vortex at a distance $r_{\perp 0}$ from the symmetry axis, the angular momentum of the system with respect to the $z$ axis must be calculated by integrating over the whole volume of the system:

$$
\langle\mathbf{L}\rangle=\int d^{3} \mathbf{r} \rho(\mathbf{r})(\mathbf{r} \times \mathbf{v}),
$$

where $\mathbf{v}$ is the superfluid velocity around the vortex and $\rho(\mathbf{r})$ is the condensate density. As discussed already, we can neglect in the TF limit the effect of the vortex on the density profile and take $\rho(\mathbf{r}) \simeq \rho_{0}(\mathbf{r})$. Then, using cylindrical coordinates around the $z$ axis, we have $\rho_{0}(\mathbf{r})=\rho_{0}\left(r_{\perp}, z\right)$. In the present geometry, $\mathbf{L}$ is parallel to the axis of rotation and its magnitude is given by

$$
\left\langle L_{z}\right\rangle=\int d^{3} \mathbf{r} \rho_{0}\left(r_{\perp}, z\right) \hat{\mathbf{z}} \cdot(\mathbf{r} \times \mathbf{v}) .
$$

The triple scalar product can be written as $\hat{\mathbf{z}} \cdot(\mathbf{r} \times \mathbf{v})$ $=r_{\perp} v_{\varphi}$, where $v_{\varphi}$ is the tangential component of the velocity. Assuming that the boundaries of the system are given by $-R_{z} \leqslant z \leqslant R_{z}, 0 \leqslant r_{\perp} \leqslant R_{\perp}$, then

$$
\left\langle L_{z}\right\rangle=\int_{-R_{z}}^{R_{z}} d z \int_{0}^{R_{\perp}} \rho_{0}\left(r_{\perp}, z\right) r_{\perp} d r_{\perp} \int_{0}^{2 \pi} v_{\varphi} r_{\perp} d \varphi .
$$

It can be easily seen that, for fixed $r_{\perp}$ and $z$, the angular integral is a line integral around a closed path, which corresponds to the quantization of the circulation (8). Therefore, the angular integral in Eq. (20) will contribute only when the closed contour encloses the vortex core. That is,

$$
\int_{0}^{2 \pi} v_{\varphi} r_{\perp} d \varphi=\oint_{r_{\perp}, z} \mathbf{v} \cdot d \mathbf{l}=\frac{\hbar \kappa}{m} 2 \pi \theta\left(r_{\perp}-r_{\perp 0}\right),
$$

where $\theta\left(r_{\perp}-r_{\perp 0}\right)$ is the step function. Remarkably, the detailed form of the velocity field around the vortex drops out in this expression. In particular, the mirror vortex contributing in Eq. (12), does not contribute to this integral at all, because it is positioned outside the condensate and therefore never enclosed by the integration contour in Eq. (21). This leads to the following result for the angular momentum:

$$
\left\langle L_{z}\right\rangle=\int_{-R_{z}}^{R_{z}} d z \int_{r_{\perp 0}}^{R_{\perp}} \frac{2 \pi \hbar \kappa}{m} r_{\perp} \rho_{0}\left(r_{\perp}, z\right) d r_{\perp} .
$$

Due to the step function, only the part of the Bose condensate at $r_{\perp} \geqslant r_{\perp 0}$, that is outside the smallest circle around the 
$z$ axis, which still encloses the vortex line, contributes to the total angular momentum and therefore to the moment of inertia of the condensate. ${ }^{2}$

We will consider a large cylindrical condensate, which provides a good approximation for very elongated cigarshaped traps in the Thomas-Fermi regime. Inserting in Eq. (22) the Thomas-Fermi density (4), it follows that the angular momentum per particle is

$$
\left\langle l_{z}\right\rangle=\frac{\left\langle L_{z}\right\rangle}{N}=\hbar \kappa\left[1-\left(\frac{r_{\perp 0}}{R_{\perp}}\right)^{2}\right]^{2} .
$$

For fixed $R_{\perp}$, it is independent of $N$. It is worth stressing that Eq. (23) is the dominant term of the angular momentum, where corrections of order $\left(\xi / R_{\perp}\right)^{2}=\left(a_{\perp} / R_{\perp}\right)^{4}$ are neglected. Equation (23) shows that even though the circulation (8) around each vortex is quantized in units of $\hbar / M$, the angular momentum per particle around the $z$ axis is not quantized in units of $\hbar$, in general.

Since $\mathbf{L}$ is linear in the velocity field, contributions from additional vortices are additive and the angular momentum per particle (23) can be generalized to a vortex array of $\kappa$ singly quantized vortices at $\mathbf{r}_{\perp 0 i}(i=1 \ldots \kappa)$ as

$$
\left\langle l_{z}\right\rangle=\hbar \sum_{i=1}^{\kappa}\left[1-\left(\frac{r_{\perp 0 i}}{R_{\perp}}\right)^{2}\right]^{2}
$$

It is interesting to note, that for a singly quantized vortex $(\kappa=1),\left\langle l_{z}\right\rangle$ is equal to $\hbar$ only when the vortex line is centered at the $z$ axis. Otherwise $\left\langle l_{z}\right\rangle$ is strictly less than $\hbar$ and decreases when the position of the vortex core moves away from the center and approaches the boundary of the condensate. When the vortex core reaches the edge of the condensate $\left(r_{\perp 0} \simeq R_{\perp}\right)$ then $\left\langle l_{z}\right\rangle \simeq 0$. Analogously, when $\kappa$ singly quantized vortices are present, the angular momentum per particle is lower than $\kappa \hbar$, unless all cores are along the $z$ axis [3]. But this configuration corresponds to one vortex with circulation $\kappa \hbar / M$, which is unstable when $\kappa>1$ [17], and breaks into an array of $\kappa$ vortices all with unit quantization $\hbar / M$.

Let us now calculate the angular momentum per particle in the simplest vortex arrays in cylindrical traps rotating with angular velocity $\Omega$. They are created if the array is permitted to reach a state of relative equilibrium minimizing the energy

\footnotetext{
${ }^{2}$ The expression (22) can be generalized to take into account a more realistic three-dimensional vortex line. Real axially symmetric traps are not cylindrical, but provide also a longitudinal harmonic confinement $\left(\omega_{z} \neq 0\right)$. It induces an inhomogeneity and a density gradient of the condensate along the longitudinal direction that will affect the velocity. The resulting vortex line must then deform along its length $[15,16]$ and meet the boundary of the condensate at a right angle in order to satisfy the physical conditions for the velocity field. Therefore, the distance vector of a quantized vortex from the $z$ axis in general depends on $z: r_{\perp 0}=r_{\perp 0}(z)$. By taking as a limit of radial integration $r_{\perp 0}(z)$ in Eq. (22), the angular momentum expression (22) allows us to take into account such more complicated vortex configurations.
}

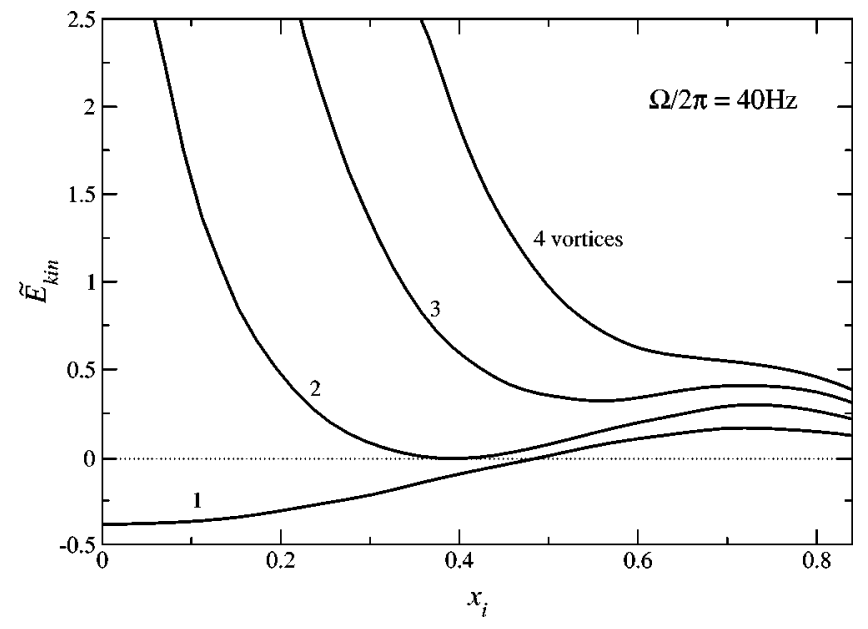

FIG. 2. Dimensionless energy of symmetrical vortex arrays of 1, 2,3 , and 4 singly quantized vortices (from bottom curve to top curve, respectively) in a trap rotating with frequency $\Omega / 2 \pi=40 \mathrm{~Hz}$, as a function of the rescaled distance of the vortex lines from the $z$ axis. The condensate has $N=2.5 \times 10^{5}$ atoms of ${ }^{87} \mathrm{Rb}$ with $R_{\perp}$ $=3.8 \mu \mathrm{m}$, which correspond to the experimental parameters of Ref. [3].

$$
E_{k i n}(\Omega)=E_{k i n}(0)-\Omega\left\langle L_{z}\right\rangle .
$$

Here $E_{k i n}(0)$ is the kinetic energy (13) of the vortex array in the nonrotating trap. As before we consider symmetrical arrays of 1-4 vortices. In Fig. 2 the kinetic energy (25) is plotted against the common normalized distance of the vortices from the $z$ axis, for an angular velocity $\Omega / 2 \pi=40 \mathrm{~Hz}$, which is, in our idealized two-dimensional trap and within the Thomas-Fermi approximation, the critical frequency where the two-vortex array first becomes stable. The corresponding critical frequencies for the symmetrical 1,3 , and 4 vortex arrays are, respectively $\Omega_{c}=33.6,43.3$, and $46 \mathrm{~Hz}$, where we have chosen for the sake of concreteness, the values of $M, R_{\perp}$ according to the experiment described in Ref. [3]. As expected, the critical frequency is larger for large vortex arrays. These values are different from and much lower than the measured critical rotation frequencies at which vortices or vortex arrays are first observed to appear, because of the existence of energy barriers, which must be overcome before the relative energy minima, formed by the vortex states, can be reached.

It can be seen that in the rotating trap, for sufficiently high values of $\Omega$, a single vortex is in relative or absolute equilibrium only at the rotation axis, as is, of course well-known, while the symmetrical arrays of vortices have equilibria at finite distances from the $z$ axis, which increase with the number of vortices because of their mutual repulsion. In Fig. 3 we plot for the same vortex arrays at the corresponding equilibrium configuration, the average angular momentum per particle as a function of $\Omega$. With increasing $\Omega$, the equilibrium positions of the vortices in the arrays move towards the $z$ axis and the average angular momentum therefore increases. For a single vortex, since the stable position is at the trap center independently of the rotation frequency, the angular momentum is always $1 \hbar$. 


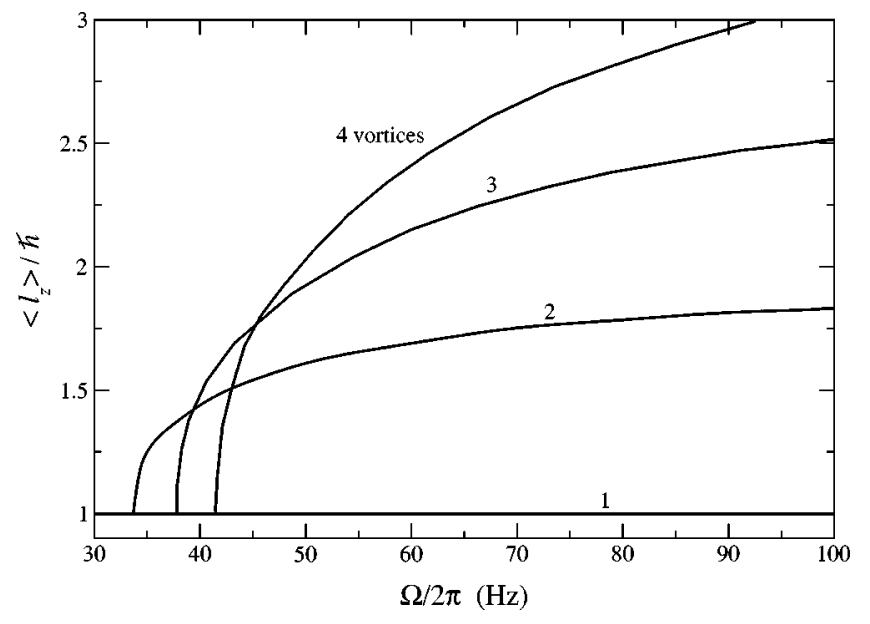

FIG. 3. Average angular momentum per particle of symmetrical arrays of $1,2,3$, and 4 vortices (from bottom curve to top curve, respectively) in relative equilibrium in a trap rotating with frequency $\Omega / 2 \pi$ as a function of $\Omega$. The values of $M$ and $R_{\perp}$ are the same as in Figs. 1 and 2.

Let us remark here that, in the absence of dissipation, vortices created experimentally in rotating traps, in general, need not correspond to minima of Eq. (25). A dissipative mechanism must be active on the scale of the time-interval during which the rotation-frequency is switched on for their positions to be able to relax to an energy minimum. After the rotation is switched off, the same mechanism will tend to lead to a relaxation of $r_{\perp 0}$ towards the minimum at the boundary $r_{\perp 0}=R_{\perp}$ of the energy (13), which increases the observed value of $r_{\perp 0}$. In the experiments on vortices in traps reported so far, dissipation seems to play a negligible role. In the absence of such a mechanism, however, the vortices with distances $r_{\perp 0}$ outside the energy minimum cannot relax but experience a force and a corresponding Magnus deflection already in the rotating trap which leads to a rotation of an array of equidistant vortices around the $z$ axis, even in the frame rotating with the trap. Vortices that are created at the boundary and have not yet reached their equilibrium distance from the $z$ axis, will then be observed to have an angular momentum, which is smaller than the equilibrium value shown in Fig. 3. From this point of view, the fact that the first vortex, which is formed, is always observed to be in the center, seems to indicate that the first vortex is, in fact, not created by the motion of a vortex line from the boundary to the center, but by a different mechanism, like, e.g., the condensation of collective excitations with $l=1$ into a vortex state with $l=1$.

Let us now turn to a discussion of some related experimental results, which have been obtained in Refs. [2,3]. From the transverse absorption images of Ref. [2], we estimate the angular momentum corresponding to the experimental configurations of the condensate with 1 up to 4 vortices. We proceed as in Ref. [3], first we obtain a qualitative measure of the ratio between the distance of each vortex from the center and the average radius of the expanding condensate, by measuring them in the portrayed images of the condensate after the time-of-flight. During the expansion the transverse lengths scale by the same factor [2,3], therefore the relation between the distance of a vortex from the center and the average radius of the condensate $\left(r_{\perp 0} / R_{\perp}\right)$ will be the same as before the expansion. From the transverse absorption images it can also be seen, that vortex arrays have uniform spatial distribution and thus, vortices are equidistant from the center, as has been assumed in Figs. 2 and 3. Then, with the values of $r_{\perp 0} / R_{\perp}$ extracted from Ref. [2] by using Eq. (24), the following estimates for the angular momentum per particle are obtained: $\left\langle l_{z}\right\rangle / \hbar=1,1.33,1.36$, and 1.38 , corresponding to the experimental configurations with $1,2,3$, and 4 vortices, respectively. These values of $\left\langle l_{z}\right\rangle / \hbar$ are smaller than the equilibrium values given in Fig. 3 , which seems to indicate that the relative radii of the vortex arrays measured in Ref. [2] are larger than their calculated equilibrium values in the rotating trap. It would be nice to compare in detail the values of $\left\langle l_{z}\right\rangle / \hbar$ we calculate from the images in Ref. [2] with those measured directly in [3]. Unfortunately, however, the experimental parameters are slightly different in Refs. [2] and [3], which makes a comparison difficult. But it is worth stressing that the calculated result for the angular momentum qualitatively agrees with the experimental data in Ref. [3], after the first jump of $\hbar$ corresponding to the nucleation of a singly quantized vortex centered in the trap, the angular momentum increases continuously when the number of vortices in the array increases [18] (which is equivalent to having increased the stirring frequency) without presenting other jumps of order $\hbar$.

\section{ENERGY SPLITTING}

The presence of a quantized vortex in a confined condensate breaks time-reversal symmetry, this produces a frequency shift of the modes with azimuthal quantum number $\pm|m|$, which in the absence of vortices, are degenerated. We want to calculate the frequency splitting of the low-lying modes in large systems due to an off-center vortex.

Theoretical calculations of the frequency shift produced by a centered vortex have been performed within different approaches: a sum-rule approach [5], a semiclassical approach based on a large $N$ expansion [6], a hydrodynamic approach [7], and a full numerical solution of the linearized equations of motion [19].

Current experiments in ENS [3] have excited the two transverse quadrupole modes $m= \pm 2$ of a quasicylindrically symmetric condensate. Due to the presence of a vortex, there exists a lift of degeneracy between the frequencies of these two quadrupole modes that causes a procession of the system. Measuring the frequency of procession, and from the analytical expressions of the frequency shift [5], the angular momentum of the system is inferred.

For simplicity, we will assume a large condensate trapped in a cylindrically symmetric trap (3), and we will study the energy splitting for the low-lying modes due to an off-center vortex at a distance $r_{0}$ from the symmetry axis. In the following, we shall use the perturbative approach $[7,16]$. (The perturbative result for the frequency splitting of the $m=$ \pm 2 modes, induced by an off-centered vortex in a trap with different symmetry from the one considered here, was re- 
ported in Ref. [20].) Linearizing the Gross-Pitaevskii equation around the condensate, using the decomposition (1) of $\Psi$ in condensate density and phase, and assuming the Thomas-Fermi approximation and the long-wavelength limit, it follows in the coupled equations,

$$
\begin{gathered}
i \omega \delta \rho=\nabla \cdot\left(\mathbf{v}_{0} \delta \rho\right)+\frac{\hbar}{M} \nabla \cdot\left(\rho_{0} \nabla \delta S\right), \\
i \omega \delta S=\mathbf{v}_{0} \cdot \nabla \delta S+\frac{g}{\hbar} \delta \rho,
\end{gathered}
$$

where $\delta \rho$ and $\delta S$ are small deviations from the equilibrium values of the density and phase, respectively. Solving Eq. (27) for $\delta S$ to first order in $\mathbf{v}_{0}$ and inserting it in Eq. (26) to eliminate $\delta S$, yields the perturbed wave equation

$$
\begin{aligned}
\left(\omega^{2}+\frac{g}{M} \boldsymbol{\nabla} \cdot \rho_{0} \boldsymbol{\nabla}\right) \delta \rho= & -i \omega \boldsymbol{\nabla} \cdot\left(\mathbf{v}_{0} \delta \rho\right) \\
& +\frac{i g}{\omega M} \boldsymbol{\nabla} \cdot\left[\rho_{0} \boldsymbol{\nabla}\left(\mathbf{v}_{0} \cdot \boldsymbol{\nabla} \delta \rho\right)\right] .
\end{aligned}
$$

In first-order perturbation theory, the shift of the eigenvalue $\omega^{2}$ is obtained from the expectation value of the perturbation, taken with the unperturbed solution of Eq. (28), $\omega_{0}^{2}$, with $\mathbf{v}_{0}=0$ :

$$
\omega^{2}-\omega_{0}^{2}=-i \omega_{0}\left\langle\mathbf{v}_{0} \cdot \boldsymbol{\nabla}\right\rangle+\frac{i g}{\omega_{0} M}\left\langle\left(\boldsymbol{\nabla} \cdot \rho_{0} \boldsymbol{\nabla}\right)\left(\mathbf{v}_{0} \cdot \boldsymbol{\nabla}\right)\right\rangle .
$$

The expectation value can be evaluated using the unperturbed Thomas-Fermi density (4), if care is taken to interpret the radial part of the space integral as a principal-value integral at $r_{\perp}=r_{0}$, because the actual condensate density, at the position of the vortex, strictly vanishes.

Let us evaluate the expectation value for a single offcentered vortex (10) in the particular case of surface modes, for which [21]

$$
\delta \rho=\sqrt{\frac{m+1}{\pi}} \frac{r_{\perp}^{m}}{R_{\perp}^{m+1}} e^{i m \varphi}, \quad \omega_{0}=\sqrt{m} \omega_{\perp},
$$

where we take $m$ first as positive. We obtain after some calculation (and using to considerable advantage the technique referred to in the first footnote) the following result for the frequency shift, due to the off-centered vortex, exhibited by the surface mode with positive $m$

$$
\omega-\omega_{0}=\frac{(m+1)}{M R_{\perp}^{2}}\left(1-\frac{r_{0}^{2 m-2}}{R_{\perp}^{2 m-2}}\right) .
$$

It turns out during the calculation that the mirror-vortex actually makes no contribution to the frequency shift. Because the velocity field enters linearly, the frequency shift induced by several vortices is additive. Replacing $m$ by $-m$ is equivalent to inverting $\mathbf{v}_{0}$, i.e., $\omega-\omega_{0}$ simply changes sign. The frequency splitting between the two frequencies (with $m$ and $-m$ ) is then

$$
\omega_{+}-\omega_{-}=\frac{2 m}{M R_{\perp}^{2}}\left(1-\frac{r_{0}^{2 m-2}}{R_{\perp}^{2 m-2}}\right) .
$$

It can be seen from Eq. (31) that there is no frequency shift for the dipole mode $m=1$, at least not to first order in $\mathbf{v}_{0}$, i.e., the Kohn theorem, which predicts $\omega(m=1)=\omega_{\perp}$, is duely respected.

The splitting of the quadrupole excitations $(m=2)$ has been measured in the ENS experiment [3]. For this case it follows from Eq. (32),

$$
\omega_{+}-\omega_{-}=\frac{6}{M R_{\perp}^{2}}\left[1-\left(\frac{r_{0}}{R_{\perp}}\right)^{2}\right] .
$$

From Eq. (33), one can see that there is a term in the frequency shift that depends on the distance of the off-center vortex from the center. It means that the procession of the eigenaxis of the quadrupole mode, $\dot{\theta}=\left(\omega_{+}-\omega_{-}\right) / 2|m|$ that is measured in the ENS experiment [3], will be slightly different for an off-center than for a centered vortex. And thus, from this difference, the distance of the off-centered vortex can be inferred.

It is worth noting that Eq. (33) in the particular case of a centered vortex $\left(r_{0} / R_{\perp}=0\right)$ does not give the same dependence on the trap and condensate parameters as the result obtained in Refs. $[5,16]$ because the symmetry of the trap considered in both calculations is different.

The preceding calculation can be extended to axial vortex modes [22] traveling along the axis of the trap with wave number $k$. For small $k$, the unperturbed modes without vortex, to leading order in $\left(k R_{\perp}\right)^{2}$, take the form

$$
\begin{aligned}
\delta \rho\left(r_{\perp}, \varphi, z\right)= & \sqrt{\frac{m+1}{\pi}}\left[1+\frac{k^{2}}{4(m+2)}\left(r_{\perp}^{2}-\frac{m+1}{m+2} R_{\perp}^{2}\right)\right] \\
& \times \frac{r_{\perp}^{m}}{R_{\perp}^{m+1}} e^{i m \varphi} e^{ \pm i k z}
\end{aligned}
$$

with frequencies

$$
\omega_{0}=\sqrt{m} \omega_{\perp}\left(1+\frac{k^{2} R_{\perp}^{2}}{4 m(m+2)}\right) .
$$

Using Eqs. (34) and (35) in Eq. (29), we obtain, after the evaluation of the expectation values to leading order in $\left(k R_{\perp}\right)^{2}$, 


$$
\begin{aligned}
\omega_{+}-\omega_{-}= & \left(\omega_{+}-\omega_{-}\right)_{0}+\frac{\hbar k^{2}}{2 M m(m+2)^{2}}\left[m^{2}+m+2\right. \\
& +4 m\left(\frac{r_{0}}{R_{\perp}}\right)^{2 m+2}-2(m+1)^{2}(m+2)\left(\frac{r_{0}}{R_{\perp}}\right)^{2 m} \\
& \left.+(m+1)\left(2 m^{2}+3 m+2\right)\left(\frac{r_{0}}{R_{\perp}}\right)^{2 m-2}\right]
\end{aligned}
$$

where $\left(\omega_{+}-\omega_{-}\right)_{0}$ is the result following from Eq. (29) to which Eq. (36) reduces for $k=0$. For the special case of a centered vortex $r_{0}=0$, and taking $m=1$, the result (36) reduces to a result derived previously in Ref. [7].

Let us now compare these results with those following from an application of the sum-rule approach, developed for this problem in Ref. [5]. As is explained there, the sum-rule approach is based on the assumption that the expectation values of the commutators of certain adequately chosen excitation operators, taken in the ground state containing the vortex, will be exhausted by the two modes $\omega_{+}, \omega_{-}$, whose splitting is to be calculated. As has been shown in Ref. [5], this approach works very well (i.e., its basic assumption is satisfied to the required accuracy) for the axially symmetric case of a centered vortex, where in the Thomas-Fermi and long-wavelength limit, the result can be checked by perturbation theory [16]. However, we shall now see that, for the case of off-centered vortices, the sum-rule approach fails, because the result of first-order perturbation theory is not reproduced.

First, we briefly recall the sum-rule approach. Let $F_{ \pm}$ $=\sum_{j=1}^{N} f_{ \pm}\left(\mathbf{r}_{j}\right)$ be the mutually adjoint operators, carrying opposite angular momentum, which excite the collective modes $\pm|m|$, respectively. Let $m_{p}^{ \pm}$be the $p$-energy weighted moments of the dynamic structure factor associated to the excitation operators $F_{ \pm}$, which can be written as expectation values of commutators between the many-body Hamiltonians of the system $(H)$ and $F_{ \pm}$:

$$
\begin{gathered}
m_{1}^{+}=\left\langle 0\left|\left[F_{-},\left[H, F_{+}\right]\right]\right| 0\right\rangle=\frac{N \hbar^{2}}{M}\left\langle\left. 0|| \nabla f_{+}\right|^{2} \mid 0\right\rangle \\
m_{2}^{-}=\left\langle 0\left|\left[\left[F_{-}, H\right],\left[H, F_{+}\right]\right]\right| 0\right\rangle=N\left\langle 0\left|\left[j_{-}, j_{+}\right]\right| 0\right\rangle,
\end{gathered}
$$

where $|0\rangle$ may be a vortex free or a vortex state, and $j_{ \pm}$ $=(\hbar / m) \boldsymbol{\nabla} f_{ \pm} \cdot \mathbf{p}$. Assuming that the moments are exhausted by the modes $\pm|m|$, the shift of the collective frequencies in the TF limit can be calculated as

$$
\hbar\left(\omega_{+}-\omega_{-}\right)=m_{2}^{-} / m_{1}^{+}
$$

Applying this prescription, let us start with the transverse quadrupole modes $m= \pm 2$. The excitation operators carrying angular momentum $m= \pm 2$, are

$$
f_{ \pm}=(x \pm i y)^{2} \text {. }
$$

Evaluating the moments Eqs. (37) and (38), and from Eq. (39), the following result for the frequency shift for the $m$ $= \pm 2$ modes, is obtained [5]:

$$
\omega_{+}-\omega_{-}=\frac{2}{M} \frac{\left\langle l_{z}\right\rangle}{\left\langle r_{\perp}^{2}\right\rangle} .
$$

Neglecting the microscopic details of the vortex core structure in the density profile, the square radii of the condensate can be evaluated using the TF approximation [11]. This prescription can be applied to a centered- or noncentered vortex. What changes in each of these configurations is the averaged value of the axial component of the angular momentum $\left\langle l_{z}\right\rangle$. Using the results obtained in the previous section, Eq. (23), the sum-rule result for the frequency splitting due to an offcenter vortex at a distance $r_{0}$ from the symmetry axis, is

$$
\omega_{+}-\omega_{-}=\frac{6}{M R_{\perp}^{2}}\left[1-\left(\frac{r_{0}}{R_{\perp}}\right)^{2}\right]^{2},
$$

where we recall that the square radius in the transverse direction of a cylindrical condensate in the TF limit is

$$
\left\langle r_{\perp}^{2}\right\rangle=\frac{R_{\perp}^{2}}{3}=\frac{4}{3}\left(a N_{\perp}\right)^{1 / 2} a_{\perp}^{2} .
$$

The splitting (42) differs from the perturbative result (33) by the factor $\left[1-\left(r_{0} / R_{\perp}\right)^{2}\right]$ and therefore systematically underestimates the frequency shift, except for a centered vortex $r_{0}=0$.

Let us try the sum-rule approach also on the calculation of the splitting of axial helical vortex modes [22] with low multipolarity and small wave number $k$. The excitation operators of the axial helical vortex modes with wave number $k$ and angular distortion $m= \pm 1$ and $m= \pm 2$ are

$$
\begin{aligned}
& f_{ \pm}=(x \pm i y) e^{ \pm i k z}, \\
& f_{ \pm}=(x \pm i y)^{2} e^{ \pm i k z}
\end{aligned}
$$

respectively. The calculation of the corresponding moments leads to the following frequency shifts. For the $m= \pm 1$ helical vortex mode,

$$
\omega_{+}-\omega_{-}=\frac{k^{2}}{M}\left\langle l_{z}\right\rangle,
$$

and for $m= \pm 2$

$$
\omega_{+}-\omega_{-}=\frac{2\left\langle l_{z}\right\rangle}{M\left\langle r_{\perp}^{2}\right\rangle}\left[1+\frac{k^{2}}{16}\left\langle r_{\perp}^{2}\right\rangle\right],
$$

where we have used $\left\langle r_{\perp}^{4}\right\rangle=3 / 2\left\langle r_{\perp}^{2}\right\rangle^{2}$. Both expressions agree with the perturbative result for centered vortices $r_{0}=0$, but fail again to reproduce the correct first-order results for the off-center vortices. 


\section{SUMMARY}

We have calculated the angular momentum of a large cylindrical condensate in the presence of an off-axis vortex line and also vortex arrays, within the Thomas-Fermi approximation. In addition, the interaction energy of vortex lines in the spatially inhomogeneous condensate has been derived for the same case, including the effects of the image vortices created by the boundary condition of vanishing normal velocity. It is interesting that the image vortices do not contribute to the angular momentum even though they strongly modify the velocity field around any off-center vortex line. The kinetic energy, on the other hand, is influenced by the presence of the image vortices.

We have seen that the contribution of a given vortex to the angular momentum per particle, decreases as it moves away from the center of the system, which leads to a smooth increase of the angular momentum as a new vortex appears at a large distance from the axis of rotation. We have obtained an estimate of the angular momentum corresponding to different experimentally observed vortex configurations [2], that qualitatively agree with the continuous behavior measured from experimental data [3]. Finally, we have studied the frequency splitting of low-lying collective modes due to the presence of an off-centered vortex, including axial vortex waves, using the perturbative approach [16] and also the sum-rule approach [5]. A comparison of the results shows, that the simple extension of the sum-rule approach to offaxis vortices, does actually not work, i.e., the sum rules are in this case, not exhausted by the two nearly degenerate modes of interest.

\section{ACKNOWLEDGMENTS}

We thank A. Csordás for useful discussions. M.G. thanks the ESF for financial support and the Theoretische Physik group at the Universität GH Essen for the warm hospitality. We would like to thank a referee for alerting us to the doubtfulness of the sum-rule approach in the case of off-axis vortices.

Note added: Recently, we have received a copy of a related work [23] reporting numerical calculations of critical frequencies and angular momentum of vortex configurations with different vorticity in an elongated trap. The vortices numerically obtained in Ref. [23] are not rectilinear but with deformed shape, and the longitudinal deformation of these vortices is responsible for the almost continuous increase of the angular momentum with respect to the angular frequency.
[1] M. R. Matthews, B. P. Anderson, P. C. Haljan, D. S. Hall, C. E. Wieman, and E. A. Cornell, Phys. Rev. Lett. 83, 2498 (1999).

[2] K. W. Madison, F. Chevy, W. Wohlleben, and J. Dalibard, Phys. Rev. Lett. 84, 806 (2000).

[3] F. Chevy, K. W. Madison, and J. Dalibard, Phys. Rev. Lett. 85, 2223 (2000).

[4] R. J. Donnelly, Quantized Vortices in Helium II (Cambridge University Press, Cambridge, England,1991).

[5] F. Zambelli and S. Stringari, Phys. Rev. Lett. 81, 1754 (1998).

[6] S. Sinha, Phys. Rev. A 55, 4325 (1997).

[7] A. A. Svidzinsky and A. L. Fetter, Phys. Rev. A 58, 3168 (1998).

[8] P. O. Fedichev and A. E. Muryshev, e-print cond-mat/0004264.

[9] A. L. Fetter, Phys. Rev. A 138, 429 (1965).

[10] G. B. Hess, Phys. Rev. 161, 89 (1967).

[11] F. Dalfovo, S. Giorgini, L. P. Pitaevskii, and S. Stringari, Rev. Mod. Phys. 71, 463 (1999).

[12] E. Lundh and P. Ao, Phys. Rev. A 61, 063612 (2000).
[13] B. Jackson, J. F. McCann, and C. S. Adams, Phys. Rev. A 61, 013604 (1999).

[14] A. L. Fetter, J. Low Temp. Phys. 113, 189 (1998).

[15] Y. Castin and R. Dum, Eur. Phys. J. D 7, 3999 (1999).

[16] A. A. Svidzinsky and A. L. Fetter, Phys. Rev. A 62, 063617 (2000).

[17] E. M. Lifshitz and L. P. Pitaevskii, Statistical Physics, Part 2 (Pergamon, Oxford, 1980).

[18] D. A. Butts and D. S. Rokhsar, Nature (London) 397, 327 (1999).

[19] R. J. Dodd et al., Phys. Rev. A 56, 587 (1997).

[20] A. L. Fetter and A. A. Svidzinsky, J. Phys.: Condens. Matter 13, R135 (2001).

[21] S. Stringari, Phys. Rev. Lett. 77, 2360 (1996).

[22] L. P. Pitaevskii, Zh. Éksp. Teor. Fiz. 40, 646 (1961) [Sov. Phys. JETP 13, 451 (1961)].

[23] J. J. García-Ripoll and V. M. Pérez-García, Phys. Rev. A 63, 041603(R) (2001). 